\section{Experiencia de mujeres con cesárea en Uruguay: el derecho a estar acompañada por una persona de su elección y las dificultades en su cumplimiento}

\author{
Women's experience with cesareans in Uruguay: \\ the woman's right to be accompanied by a \\ person of her choice and difficulties \\ with enforcement
}

\section{Experiência de mulheres com cesariana no Uruguai: o direito a estar acompanhada por uma pessoa de sua confiança e as dificuldades no seu cumprimento}

\section{Resumen}

El objetivo de este artículo es analizar el acompañamiento durante la cesárea, por parte de una persona de confianza de la mujer, consagrado por la Ley no 17.386 de "acompañamiento en el parto", aprobada en Uruguay en el año 2001. Los resultados forman parte de una investigación mayor sobre la vivencia y el significado de la cesárea para las mujeres que pasaron por esta experiencia. Para ello se optó por una metodología cualitativa de carácter exploratorio y descriptivo. La técnica utilizada fue la entrevista en profundidad, realizada a un total de 31 mujeres, cuyas cesáreas tuvieron lugar en su primera gestación, en Montevideo y área metropolitana (Uruguay). De los resultados obtenidos, se concluye que el acompañamiento en el proceso de trabajo de parto y nacimiento es vivido por las mujeres como un factor de protección emocional ante las ansiedades que genera la experiencia de una cirugía mayor como es la cesárea. Sin embargo, para las mujeres entrevistadas, el "acompañamiento" más que continuo se caracterizó por una serie de separaciones, tanto de sus acompañantes, como de sus hijos/as, lo cual provocó ansiedad, angustia, sentimientos de ambivalencia ante el recién nacido, dificultades en el establecimiento del vínculo materno-filial, sobre todo en el puerperio inmediato.

Salud Reproductiva; Cesárea; Trabajo de Parto
Carolina Farias 1

Alejandra López Gómez ${ }^{1}$

doi: 10.1590/0102-311X00176816

\author{
Correspondencia \\ C. Farías \\ Facultad de Psicología, Universidad de la República. \\ República 1667, Montevideo 11200, Uruguay. \\ cfarias@psico.edu.uy \\ 1 Facultad de Psicología, Universidad de la República, \\ Montevideo, Uruguay.
}




\section{Introducción}

El artículo presenta los resultados de la investigación Vivencias y Significados de la Cesárea para las Mujeres que Pasaron por la Experiencia, en particular aquellos relativos al acompañamiento por parte de una persona de confianza de la mujer, durante el trabajo de parto y nacimiento, al amparo de la Ley no 17.386 , aprobada en Uruguay en 2001. Se analiza las experiencias de acompañamiento durante la cesárea y el nacimiento que relatan las mujeres y las dificultades que vivieron durante el tiempo de hospitalización.

La elección del tema se basa en la relevancia que tiene para la agenda de los derechos reproductivos de las mujeres. Desde las reivindicaciones del movimiento de mujeres y feminista de la década de los 1960 hasta los 1990 2,3, mucho se ha profundizado sobre distintos aspectos involucrados en los procesos y decisiones (no) reproductivas de las mujeres: anticoncepción, maternidad elegida, aborto, atención al embarazo, parto y puerperio, menopausia, entre otros. Sin embargo, el alto índice de cesárea registrado a nivel nacional, internacional $4,5,6,7,8,9$ ha generado que distintos actores (organismos internacionales, organizaciones sociales, profesionales, gobiernos, academia) colocaran este asunto en la agenda pública como un problema de salud y de derechos humanos. El énfasis en producir conocimiento desde las propias mujeres que viven la experiencia es sólo el punto de partida. Se trata de un asunto complejo, donde intervienen múltiples factores de manera interconectada.

Desde 1985, la Organización Mundial de la Salud (OMS) sugiere que "no hay justificación en ninguna zona geográfica para tener más de 10-15\% de cesáreas" 4, lo que ha sido ratificado en 2015 5. En Uruguay, la cesárea alcanza un promedio nacional de casi $50 \%, 33,4 \%$ se producen en el subsector público y 55,3\% en el subsector privado de atención en salud, según datos proporcionados por el Sistema de Información Perinatal para Uruguay el año 2014. Sin embargo, tal es la naturalización de esta cirugía que no se cree necesario informar sobre los efectos físicos, psíquicos y emocionales.

En este sentido, la investigación buscó centrarse en las experiencias de las mujeres que transitaron una cesárea al culminar su primera gestación. Uno de los objetivos específicos fue analizar las vivencias subjetivas de hospitalización y de intervención quirúrgica, explorando las experiencias de acompañamiento durante el trabajo de parto y nacimiento por una persona de confianza de la mujer.

Uruguay ha sido pionero en la región en legislar sobre el derecho de las mujeres a estar acompañadas, durante el trabajo de parto y nacimiento de sus hijos, por una persona de su elección. La Ley no 17.3861 , aprobada en agosto de 2001 por unanimidad en ambas cámaras legislativas, expresa en su artículo 1ero: "Toda mujer durante el tiempo que dura el trabajo de parto, incluyendo el momento mismo del nacimiento, tendrá derecho a estar acompañada de una persona de su confianza o en su defecto, a su libre elección, de una especialmente entrenada para darle apoyo emocional".

Los argumentos que justificaron la aprobación de la ley se basaron en los informes sobre los beneficios para la mujer en trabajo de parto y nacimiento, elaborados por Centro Latinoamericano de Atención Perinatal, Organización Panamericana de la Salud (CLAP-OPS) y la OMS. Algunos de los argumentos a favor del acompañamiento de las mujeres por una persona de su elección estuvieron relacionados con la mejor satisfacción de las mujeres sobre la experiencia del nacimiento, menor tiempo de duración del trabajo de parto, menor necesidad de aplicación de técnicas invasivas o medicación, así como la disminución en la necesidad de analgesia durante el parto 10 , o sea que una mujer a la que se le brinda apoyo y sostén emocional, requiere menos intervenciones médicas, reduce los niveles de ansiedad y se mejora la percepción del trabajo de parto y parto 10,11,12,13.

Por otro lado, la ley, al incluir "el momento mismo del nacimiento" y no sólo el parto, habilita el acompañamiento en caso de cesárea. Este derecho no requiere el consentimiento del personal de salud tratante, sino que es inherente a la decisión de la mujer, a diferencia de otros países que legislaron en la materia, pero que el derecho es sometido al consentimiento médico en el momento del nacimiento 13 .

A pesar del tiempo transcurrido desde la aprobación de la ley, se observan dificultades en su implementación en los servicios de salud públicos y privados 13,14,15. En particular, las dificultades y barreras se incrementan cuando se trata de cesárea, tal como observamos en el estudio cuyos resultados presentamos aquí.

Para el tratamiento del problema de investigación, nos basamos en los aportes de las teorías sociales interpretativas en el campo de la salud, en articulación con aquellas que provienen del corpus teórico feminista, en particular el concepto de género y sus derivaciones en el campo de la salud, asî 
como los desarrollos sobre medicalización de la sociedad y del cuerpo de las mujeres. Este enfoque permite pensar las improntas sociales que se inscriben en la subjetividad de las mujeres, en relación a la maternidad, así como en la expropiación de los cuerpos de las mujeres-madres durante el proceso de gestación y nacimiento.

\section{Método}

Se realizó un estudio exploratorio, descriptivo y analítico, basado en una metodología cualitativa, por considerar que era la más adecuada a los objetivos propuestos. Este abordaje permite visualizar las distintas dimensiones del fenómeno en dos sentidos; por un lado, poner de relieve la voz de las mujeres, y por otro, dejar en evidencia que "las mujeres" no son un conglomerado homogéneo, sino que atraviesan por esta experiencia recurriendo a diversos mecanismos psíquicos y de apoyo social.

Se utilizó como técnica la entrevista en profundidad semiestructurada, la cual permite acceder a las experiencias significativas, a la vez que posibilita no quedarse en el discurso "políticamente correcto", sino que busca un acercamiento "denso" al sentir y pensar de las personas entrevistadas 16 . Las entrevistas fueron realizadas entre agosto y noviembre de 2014, con una duración aproximada de una hora cada una.

Se definió una muestra intencional no probabilística, en base a los siguientes criterios de inclusión: mujeres entre 20 y 40 años; primerizas (o sea, que la cesárea haya ocurrido al finalizar su primera gestación) y cuyo/a hijo/a naciera sano/a, y continuara así para el momento de la entrevista, además de que hayan transitado por embarazos normales y a término. Se excluyó a mujeres con cesárea de emergencia (es decir, que requirieron de la intervención de forma imprevista e inminente por riesgo vital materna o fetal) y mujeres con discapacidad psíquica y/o intelectual.

La muestra se dividió en dos grupos: mujeres con cesárea programada y mujeres con cesárea intraparto (con trabajo de parto) e incluyó a mujeres entre el primer mes de puerperio y hasta los 24 meses posteriores al nacimiento. Se entiende por cesárea programada aquella que debió realizarse previo al inicio del trabajo de parto por la indicación médica de interrumpir el embarazo, sin la posibilidad de intentar un parto vaginal. No se incluyó ningún caso en el que la cesárea haya sido solicitada por la única voluntad de la mujer (sin motivos médicos). Se realizó un total de 31 entrevistas a mujeres de los departamentos de Montevideo, Canelones y Maldonado. Todas las entrevistadas tenían por lo menos enseñanza secundaria completa y sus edades oscilaron entre 28 y 40 años. La investigación cubrió exclusivamente a mujeres usuarias del Sistema Nacional Integrado de Salud, tanto del sector público como privado, con mayoría de este último, en concordancia con la distribución asistencia nacional 17.

Las entrevistadas lo hicieron de manera informada y voluntaria, previa firma del consentimiento. Las mismas fueron grabadas para su transcripción, codificación y análisis mediante método inductivo, posteriormente fueron formateadas e inicialmente revisadas con el objetivo de identificar inconsistencias y/o distorsiones, que pudieran provenir tanto de los sujetos, de la entrevistadora, así como de la interacción entre sujeto-investigadora. En un segundo momento, se procedió con la técnica de análisis de contenido y a la reducción de los datos mediante la categorización y codificación del material, dividiéndolo en unidades temáticas que expresaban ideas significativas del problema de investigación que se buscaba conocer. Finalmente, se crearon categorías empíricas y operacionales que surgieron de las lecturas sucesivas del material de campo y que expresaban relaciones y representaciones previamente no consideradas. Estas categorías empíricas fueron confrontadas con las categorías analíticas previamente definidas.

El estudio contó con el aval del Comité de Ética de la Investigación de la Facultad de Psicología de la Universidad de la República, en el marco del Decreto Nacional no 379/008 sobre aspectos éticos en investigación con seres humanos.

La protección de la identidad de las entrevistadas se realizó mediante el cuidado de datos identificatorios, esta protección se aplica a cualquier reporte o comunicación de los resultados obtenidos. Del mismo modo, el manejo digital de la información fue cifrado, con el objetivo de que la información no pueda ser decodificada por nadie ajeno a la investigación. 


\section{Resultados y discusión}

El acompañamiento emocional durante el trabajo de parto y nacimiento fue reportado en varios estudios 13,18. En el caso de nuestro estudio, las mujeres relataron que de no haber estado acompañadas la experiencia hubiera sido más angustiante, dada la inseguridad y el miedo que la cesárea les producía. El acompañamiento por una persona de su elección fue vivido como un factor de protección emocional.

“(...) eso a mí me rescató bastante en esta cuestión de que yo no quería que naciera por cesárea bajo ningún concepto y entonces, esta cuestión de estar con él [su pareja] era sumamente necesaria para que todo esto que yo estaba pasando, lo pasara bien" (E1, 15 meses, intraparto).

Sin embargo, si analizamos en profundidad vemos que el acompañamiento estuvo mediado por continuas separaciones, fundamentalmente registradas en tres momentos del proceso: al llegar a la institución de salud, en la preparación/traslado al block quirúrgico y anestesia, y luego del nacimiento.

"Cuando me entraron en la urgencia él no entró conmigo, no lo dejaron entrar. Nunca, de las veces que fui a la urgencia, nunca lo dejaron entrar, eso me molestó, nunca me gustó” (E15, 10 meses, intraparto).

"No lo dejaron ir conmigo al block. Después en el momento del nacimiento lo dejaron entrar pero mientras iba no, no lo dejaron. Entonces pedí 'yo no quiero estar sola', por favor, si no viene mi pareja, 'iij no quiero estar sola!!!'. No sé, me tocó una guardia de perros, porque yo no digo que sea la institución, digo, hay cosas de la institución, pero ahi hay gente, hay personas, las parteras divinas y todo lo demás pero esa guardia... todo frío, todo impersonal, te vamos a sacar al bebé y tá y vos sos una cosa... y estás sola” (E8, 2 meses, intraparto).

Las mujeres debieron relacionarse con la institución, sin contar con la contención o apoyo de alguien de su confianza, lo cual las colocó en una situación de mayor vulnerabilidad, al tener que afrontar la intervención sin la debida contención emocional.

“(...) él no estaba, iban pasando todos los procedimientos, (...) y yo estaba pendiente que él no estaba, él no estaba. Incluso yo ya estaba acostada, se iba a comenzar el procedimiento y él todavía no estaba. Mi miedo era que sacaran a la bebé y él no esté... ahí me dijeron que él era el último, que luego entraba, ya empezada la cesárea lo dejaban entrar" (E7, 1 mes, programada).

En el caso de la cesárea, la ley vigente prevé que el acompañamiento se concrete desde el ingreso a la institución de salud. Sin embargo, todas las entrevistadas narraron que desde que salieron de la habitación hasta el comienzo mismo de la cirugía se encontraban solas, sin sus acompañantes. La aplicación de la anestesia se realizó en todos los casos sin que la mujer estuviera acompañada. La justificación que se les dio por parte del personal de la salud fue que, en ese lapso, los acompañantes se estaban preparando y que entrarían una vez aplicada la anestesia. Sin embargo, la ley no admite razones que justifiquen la separación en ningún momento, lo que debiera incluir la preparación para quirófano y la aplicación de la anestesia. Se busca así beneficiar a las mujeres y su derecho de decidir, pero en la práctica tal derecho no es plenamente respetado durante todo el proceso.

Otro elemento a considerar es el lugar físico dentro del quirófano para el acompañante. Las mujeres relatan que sus acompañantes no eran visibles para ellas, ya que muchas veces el acompañamiento es "aceptado", pero sólo como una protección de los derechos del padre de ver nacer a su hijo.

"[se le pregunta por su acompañante] no, yo no lo veía, estaba como en los pies, viendo el nacimiento" (E12, 13 meses, intraparto).

“Dónde está mi esposo?... porque pensé que iba a estar dándome la mano, que iba a estar conmigo, pero no, en [su mutualista] lo dejan... es como si estás afuera, por ejemplo, ahí está la puerta y él está parado del otro lado de la puerta. Mira en realidad (...) yo que sé, en ese momento como que me sentí sola" (E4, 21 meses, programada).

Si bien la ley fue aprobada para proteger los derechos de las mujeres de elegir una persona de su confianza durante el trabajo de parto y nacimiento, ello queda supeditado a la voluntad del personal de salud que decide el rol y el lugar físico que va a ocupar el acompañante en la sala.

Un factor relacionado con la separación o distancia que se le impone al acompañante en la sala de operaciones es su justificación en protocolos quirúrgicos, los cuales no fueron adaptados a los requerimientos de la ley, en sus ya 15 años de vigencia, como por ejemplo, el lugar para el acompañante entre los técnicos.

"Estaban a punto de cortar y [su pareja] no venía y entró y le dije que quiero que esté el padre [al lado de ella] y me dijo que no, que la cabeza es del anestesista. Pero, jdéjalo que esté a mi lado!-le dije, y me dijo: 'ya te dije que la cabeza es del anestesista”' (E13, 23 meses, urgencia). 
El derecho a estar acompañada es también vulnerado una vez que se produce el nacimiento, ya que no se le permite a la mujer permanecer con el bebé en el quirófano, por tanto debe elegir entre estar ella acompañada o que lo esté su hijo/a. Según manifestaron las entrevistadas, sus acompañantes se fueron con los bebés. Pudimos evidenciar que el acompañamiento como factor de protección emocional, estuvo más bien relacionado con la tranquilidad y la seguridad que le produjo a la mujer que alguien de su confianza estuviera con el bebé más que con ella misma.

"Estaba tranquila de que la bebé estaba en buenas manos. Yo le decía a [su pareja] 'vos ocúpate de ella, yo confío en que vos te estás ocupando de ella" (E7, 1 mes, programada).

En la cesárea, las mujeres no pueden ver el nacimiento de sus hijos, debido a uno de los campos estériles que se le coloca a la altura de pecho, por tanto, no saben del nacimiento hasta que alguien explícitamente se los comunica. En los relatos de las entrevistadas, fue el propio equipo de ginecología quién da la noticia, en otras, el acompañante. Sin embargo, observamos testimonios en que nadie les dice nada, incluso ante sus preguntas insistentes por saber si todo había salido bien.

En una cesárea, como en cualquier cirugía, el temor ante la muerte se intensifica. Ese tiempo, aunque sea breve, sin saber si nació el hijo y si se encuentra bien, genera en la mujer estrés y angustia que podría ser evitada.

"Me ponen el campo, entonces yo no lo veía. Tuve sí la sensación cuando él salió, sentí el vacío, sentí que salió el bebé, pero no lo sentía llorar, entonces ijme asusté mucho!!” (E17, 6 meses, programada).

Finalmente, por lo general, alguien del equipo de neonatología le acerca al bebé, ya vestido, antes de llevárselo del quirófano. Sobre este punto, se pueden realizar, por lo menos, dos consideraciones: por un lado, la imposibilidad para la mujer de tener contacto con el bebé, ya que sus manos fueron atadas para el acto quirúrgico y por otro, la imposibilidad del contacto piel con piel madre-bebé.

En cuanto a la primera, si no lo había percibido antes al intentar tocar al bebé la mujer se da cuenta que le han atado ambos brazos en cruz y que no podrá hacerlo. Varias de las entrevistadas se refirieron a esto como "la crucifixión".

"En la cesárea... yo pensé que yo lo podía agarrar... no, no, no, no fue... jyo estaba como crucificada!, así literalmente [solloza] con las dos manos atadas (...) Yo le digo: 'dejámela... ponémela acá, déjamela acá (...) y ahí me di cuenta que las manos las tenía atadas, que no la podía tocar pero yo pensaba: está él [su pareja], él la puede sujetar, me la puede poner acá (...) " (E14, 24 meses, programada).

Por lo general, en una cirugía, los brazos son atados para evitar que la persona los mueva y toque la cavidad intervenida. Por otro lado, también se justifica en la colocación de las vías para suministro de suero, medicación, medición de presión, etc. Sin embargo, ninguna de estas razones fue explicada a las mujeres antes de la cesárea y por tanto no pudieron optar o negociar otras formas, como por ejemplo, que las vías fueran colocadas en un solo brazo o incluso, que se las liberara una vez nacido el niño/a.

"Hay muchas de esas cosas que quedan a la discrecionalidad médica, depende del [equipo] que te toque, lo que tenga ganas de hacer... iijdeciden si podés tocar a tu hijo!!! iijes muy fuerte!!! jellos determinan si tu hijo te va a tocar inmediatamente después de que sale o no! [llora angustiada, su tono de voz es de rabia y bronca]" (E13, 23 meses, intraparto).

Habitualmente, los profesionales de la salud no sólo toman sus decisiones técnicas basados en la evidencia científica o disciplinar, sino también a partir de sus esquemas de percepciones y valores sobre el papel de las mujeres en el parto y nacimiento de sus hijos. En tanto agentes sociales reproducen las imposiciones de género 19,20, perpetuando la disciplina del cuerpo de las mujeres.

La segunda dimensión está íntimamente ligada con la anterior y se refiere a las prácticas institucionales y de "discrecionalidad", mediante las cuales se niega el contacto piel con piel de la madre y el bebé, luego del nacimiento vía cesárea. Por “contacto piel con piel” nos referimos a la práctica mediante la cual el recién nacido es colocado en contacto directo con la piel de su madre. Desde el punto de vista psíquico, Adolfo Perinat nos dice: "La psicología ha demostrado (las célebres experiencias de Harlow) que el contacto piel a piel tiene otros efectos sedantes y de equilibrio psicológico. Este es otro caso de 'expansión' de una función primitivamente biológica hacia el campo psíquico” 21 (p. 93).

Los organismos internacionales (Fondo de las Naciones Unidas para la Infancia-UNICEF-, OMS) recomiendan el contacto piel con piel de la madre y el recién nacido por razones sanitarias, debido a los beneficios en la termoregulación y la respiración, así como por ser un factor protector de la salud emocional del bebé y la madre. Es considerado uno de los mayores facilitadores de un comienzo de lactancia exitosa 22. En nuestro país, el Ministerio de Salud Pública recomienda que se generalice la 
práctica del piel con piel, incluyendo los nacimientos vía cesárea: "En caso de cesárea, el contacto piel a piel deberá realizarse inmediatamente después del nacimiento o por lo menos durante la primer hora después de que la madre haya recuperado el conocimiento, en caso de anestesia general" 22.

Sin embargo, es frecuente que la mujer y el bebé sean separados durante la recuperación anestésica, obstaculizando 23, según Perinat 21 (p. 92) "la relación psicológica que ha de establecerse entre madre-hijo".

Ninguna de las mujeres entrevistadas pudo estar con su bebé más de unos segundos mientras se lo mostraban y no les fue permitido el contacto piel con piel. Estas prácticas fueron "justificadas” por el personal de la salud en base a la temperatura ambiente en el quirófano o por los riesgos de infección. Sin embargo, ello no se sustenta en la evidencia científica, ni en la propia normativa que indica que el bebé puede permanecer con su madre sin que existan riesgos para ninguno de ellos 22 .

"Ese primer contacto inicial... por más que no haya sido un parto, en realidad no te lo tenés que perder, como que son esas cosas grandes, me parece, que nadie se las tendría por qué perder" (E19, 16 meses, programada).

"Enseguida me lo mostraron, asi que tá, no me olvido más la carita de él, nos miramos, me lo pusieron acá un poquito, me lo sacaron enseguida, en realidad se lo llevaron en seguida, me lo pusieron así como que piel con piel con la cara y tá, se lo llevaron enseguida" (E4, 21 meses, programada).

Según la información brindada, el tiempo de separación varió entre 20-30 minutos hasta 3 horas, dependiendo generalmente de los protocolos institucionales, en relación a la recuperación anestésica, la planta física o del propio personal (camilleros, enfermería).

"Enseguida pasé... mirá, él nació a las tres menos cinco y yo tres y media estaba en la habitación" (E17, 6 meses, programada).

"Eso de tener que estar una hora y media después... jyo no tenía ni idea! No tenía ni idea que eso pasaba y eso sí que fue feo, fue re-feo y yo no sé si es necesario que eso sea asî" (E7, 1 mes, programada).

Durante ese período la mujer se encuentra en "reanimación”, mientras que el bebé junto con su padre, o quien haya acompañado a la mujer, deben esperar en la nursery.

"A ella se la llevaron a nursery y a mí me dejaron sola, en el pasillo. Supuestamente, esa era la sala de 'recuperación', pero era un pasillo, donde entraba gente, salía gente, pasaban las enfermeras y me decían: 'es divina, tu bebé es divina', y yo les decía 'traémela, yo quiero estar con ella!!' (E8, 2 meses, urgencia).

El acompañamiento del recién nacido en la sala de enfermería, por parte de su padre o una persona cercana, es un cambio sustantivo en relación a las tradicionales prácticas institucionales, donde los bebés se quedaban solos y se les veía a través de un vidrio. El acercamiento del padre genera vínculos de apego con el recién nacido que beneficia el desarrollo del vínculo paterno filial 24,25,26,27,28. Sin embargo, esto no debería estar en detrimento del derecho y deseo de la mujer de permanecer junto a su hijo/a e iniciar la lactancia inmediatamente al nacimiento o dentro de la primera hora de vida.

El tiempo de separación de la madre y su bebé no informado previamente a la cesárea, lo cual incrementó el grado de ansiedad e incertidumbre, en la mayoría de las entrevistadas. De todos modos, la angustia por la separación y la soledad con que fueron vividos esos momentos, también fue experimentado por aquellas que sí tenían conocimiento de que, en caso de cesárea, estarían separadas de sus hijos/as (por lo general, quienes sabían este detalle fue porque amigas o familiares que vivieron esta experiencia se lo habían contado).

El grado de estrés generado, la ansiedad, la soledad y la angustia, son factores asociados al nacimiento por cesárea que pueden dificultar el vínculo materno-filial y el (buen) comienzo de la lactancia 5,22 , pero también la vivencia posterior del puerperio 28

No hubo justificación que haya satisfecho las necesidades de las mujeres por conocer a su hijo/a, tenerlo/a junto a ella y comenzar la lactancia. En particular, porque el contacto en el quirófano fue mínimo, algunas de ellas no lo llegaron a ver, otras no pudieron tocarlos y aquellas que sí, sólo lo hicieron por unos segundos.

Las respuestas institucionales, no evitaron que en estos casos, las mujeres pidieran que sus acompañantes o sus bebes estuvieran con ella.

"Cuando [la ginecóloga] me dijo 'no se puede quedar acá porque no puede venir con el papá para acá, porque sino hay otra gente y van a querer venir también' y tá, esa cosa de que no es para todos... pero jes un bolazo!" (E19, 16 meses, programada).

Las mujeres desconocían dónde, con quién y el estado en que estaba su hijo y eso les generaba mucha ansiedad y angustia, sobre todo en aquellas mujeres cuya cesárea fue intraparto, ya que la intervención ocurrió, en la mayoría de los casos, por algún riesgo (por ejemplo, sufrimiento fetal). 
Sin embargo, en las salas de recuperación no tenían información sobre el estado de los bebés, ni si efectivamente sus acompañantes estarían con ellos.

"Yo pensé que parte de la recuperación ella iba a estar conmigo. ¿Por qué no me la dejaban ahí? ¿Por qué no la tenía yo ahí mientras...? No sé, si no había nada especial, ¿por qué no la podía tener yo acá arriba si la herida era allá abajo y la recuperación podía ser igual? Los brazos los sentís igual, lo que no sentís es de la cintura para abajo, una podía tenerla prendida al pecho en ese momento que yo me estaba recuperando [se le quiebra la voz, solloza]” (E14, 24 meses, programada).

"Cuanto te pasan a recuperación, como que no hay motivos para que no estés vinculada con el bebé. Si tienen que armar un lugar especial para las madres y que ahí puedan estar con su bebé... jes como básico! (...) Yo estoy segura que todo esto, cada uno [refiriéndose a cada institución] hace las cosas como les sirve, no es que haya un organismo que regule, organice, vas hablando con las mujeres y cada una tuvo una experiencia totalmente distinta" (E12, 13 meses, intraparto).

En el siguiente relato, podemos observar el impacto emocional generado por la separación, pero también por la conducta de los profesionales en la sala de reanimación:

"Ahi fue fuerte, fuerte... ifuerte!, como que no podía creer que estuve dos horas y media! Primero que yo pedí por todas las posibilidades: si yo podía ir a ver a mi bebé o si el bebé podía venir... no sé, iqque lo resolvieran!! (...) y ahí pasó algo que emocionalmente... empiezo a escuchar que habia un niño grave, "hay que horrible, pobrecito, que está grave', y ahí yo me largo a llorar y decía ¿qué niño?, ¿es el bebé que acaba de nacer? 'no, no, quédate tranquila, es otro'... Pero tá, la situación era horrible: yo ahí acostada, sin poder pararme, sin sentir mis partes, sin poder darle vida al cuerpo y escuchaba a la gente que estaba ahí hablar de un bebé grave! ... Ahí me largué a llorar, no podía más... ijla impotencia en realidad!!" (E12, 13 meses, intraparto).

De las experiencias relatadas, hubo una que llamó la atención por su excepcionalidad.

"Se dio algo que en realidad por lo que hablan no es muy común, a mí me dejan en la sala de recuperación y una enfermera me lo trae (...) y me dice 'porque yo prefiero que esté contigo, yo soy de la idea de que esté con la mamá y no que ande de brazo en brazo' y tá, jme pareció bárbaro!" (E4, 21 meses, programada).

Aparece nuevamente la "discrecionalidad" de los técnicos, aunque esta vez fue en "beneficio" del bebé y la mujer. Esta mujer, ¿hubiera podido estar con su hijo si la enfermera fuera otra? ¿Le habrían dicho que no era posible?

\section{Conclusiones}

El estudio permitió analizar el acompañamiento de las mujeres durante el nacimiento vía cesárea, ofreciendo resultados novedosos en un campo poco explorado a nivel regional e internacional. En general, los estudios disponibles refieren al trabajo de parto y parto vaginal y muestran similares resultados, cuando el acompañamiento se vio obstaculizado o incluso negado 29,30,31.

El estudio reveló que para las mujeres estar acompañadas durante la cesárea fue un factor de protección emocional, al sentirse más seguras por tener a alguien cercano y de su confianza, lo que concuerda con investigaciones relacionadas al acompañamiento durante el parto 32,33 . Sin embargo, el cumplimiento por parte de los servicios y personal de la salud de la "ley de acompañamiento" vigente en Uruguay no es pleno. En este sentido, la estancia hospitalaria estuvo marcada por continuas separaciones de la mujer y su acompañante.

Por otro lado, las prácticas específicas en quirófano, entre ellas la separación madre-recién nacido, no contacto piel con piel y salida del bebé y su acompañante del quirófano, así como el tiempo que las mujeres permanecen en sala de recuperación anestésica, fueron elementos negativos referidos por las mujeres de sus experiencias de cesárea. La imposibilidad de tener contacto inmediato con el hijo recién nacido produjo en las mujeres sentimientos de angustia y frustración.

Algunas de las características referidas por las mujeres, en relación a estas separaciones, fueron la dificultad para expresar su dolor psíquico (ya que "el bebé estaba bien" y eso les tendría que bastar como madres), sentimiento de extrañeza hacia el bebé cuando se encuentran por primera vez (en general un tiempo que va desde la media hora a las tres horas, dependiendo a los protocolos institucionales sobre anestesia y recuperación), entre otras.

Si bien la ley prevé que la mujer esté acompañada en todo momento, las experiencias relatadas por las mujeres sobre el acompañamiento están atravesadas por continuas separaciones: la mujer es 
separada de su acompañante en las revisiones médicas al ingreso hospitalario, en el momento del traslado al quirófano y durante la aplicación de la anestesia. Finalmente, es separada del bebé luego del nacimiento. Al momento de nacer el niño/a, la mujer debe decidir si el acompañante se retira del quirófano con el bebé o si se queda con ella, lo que deja en evidencia la imposibilidad real de ejercer el derecho de estar acompañada. Estas experiencias vividas por las mujeres parecen responder a lógicas institucionales y a condiciones materiales que no habilitan y/o dificultan el alojamiento conjunto madre-bebé luego del nacimiento.

Cabe destacar que no se encontraron diferencias significativas en los relatos de las mujeres que fueron atendidas en el sector público, de aquellas que lo hicieron en el sector privado, en relación al tema que nos convoca, a diferencia de estudios similares en la región donde la atención en el sector privado mostró mayor disposición a respetar las solicitudes de las mujeres, entre ellas las de estar acompañada.

Desde el sistema de salud y el personal sanitario en particular, no se visualiza esta situación como problema y factor de riesgo para la salud mental de la mujer y por tanto, no se ha logrado, hasta el momento, adecuar las condiciones edilicias y/o las prácticas institucionales para dar pleno cumplimiento a lo que establece la ley vigente en Uruguay, lo cual afecta directamente el bienestar emocional de las mujeres en un momento significativo como es el nacimiento de sus hijos vía cesárea.

\section{Colaboradores}

La dos autoras contribuyeron sustancialmente en la elaboración del artículo.

\section{Agradecimientos}

La investigación se realizó en el marco de la obtención del título de Magister en Psicología Social de la Facultad de Psicología de la Universidad de la República (Uruguay) y contó con el financiamiento de la Agencia Nacional de Investigación e Innovación (ANII) en su programa de Becas para Posgrados Nacionales. 


\section{Referencias}

1. Poder Legislativo de la República Oriental del Uruguay. Ley no 17.386, de 23 de agosto de 2001. Dispónese que toda mujer durante el tiempo que dura el trabajo de parto, incluyendo el momento del nacimiento tendrá derecho a estar acompañada de una persona de su confianza o en su defecto, a su libre elección de una especialmente entrenada para darle apoyo emocional. Diario Oficial 2001; 30 ago.

2. United Nations Population Division; United Nations Population Information Network; United Nations Population Fund. Conferencia Internacional de Población y Desarrollo. http://www.un.org/popin/icpd/ newslett/94_19/icpd9419.sp/1lead.stx.html (accedido el 16/Oct/2016).

3. Naciones Unidas. Informe de la Cuarta Conferencia Mundial sobre la Mujer. Nueva York: Naciones Unidas; 1996.

4. Organización Mudial de la Salud. Tecnología apropiada para el parto. http://www.junta deandalucia.es/salud/export/sites/csalud/ga lerias/documentos/p_4_p_2_promocion_de_ la_salud/embarazo_y_salud/tecnologia_parto. pdf (accedido el 16/Oct/2016).

5. Organización Mudial de la Salud. Declaración de la OMS sobre tasas de cesárea. http://apps. who.int/iris/bitstream/10665/161444/1/ WHO_RHR_15.02_spa.pdf?ua=1 (accedido el 16/Oct/2016)

6. Odent M. La cesárea. Barcelona: Editorial La Liebre; 2006.

7. Patah L, Malik A. Modelos de assistência ao parto e taxa de cesárea em diferentes países. Rev Saúde Pública 2011; 45:185-94.

8. Programa Nacional de Salud de la Mujer y Género, Dirección General de la Salud, Ministerio de Salud Pública. Guías en salud sexual y reproductiva. Capítulo: normas de atención a la mujer en proceso de parto y puerperio. Montevideo: Ministerio de Salud Pública; 2014
9. Ministerio de Salud. Objetivos sanitarios nacionales 2020. Montevideo: Ministerio de Salud; 2015 .

10. Hodnett ED, Gates S, Hofmeyr GJ, Sakala C. Apoyo continuo para las mujeres durante el parto. Cochrane Database Syst Rev 2007; (3):CD003766.

11. Poder Legislativo de la República Oriental del Uruguay. Exposición de motivos "ley de acompañamiento". https://parlamento.gub.uy/do cumentosyleyes/ficha-asunto/15206 (accedido el 16/Oct/2016)

12. Logo C, Andraus L, Barbosa M. Participação do acompanhante na humanização do parto e sua relação com a equipe de saúde. Rev Eletrônica Enferm 2010; 12:386-91.

13. Dodou H, Rodrigues D, Guerreiro E, Guedes E, Lago P, Mesquita N. La contribución del acompañante para la humanización del parto y el nacimiento: la percepción de las púerperas. Esc Anna Nery Rev Enferm 2013; 18:262-9.

14. Mujer y Salud en Uruguay. Observatorio nacional en género y salud sexual y reproductiva en Uruguay. Informe 2008. Montevideo: Mujer y Salud en Uruguay; 2008.

15. Mujer y Salud en Uruguay. Avanzar sobre lo logradoEstado de situación de la salud y los derechos sexuales y reproductivos en Uruguay. Monitoreo 2010-2014. Montevideo: Mujer y Salud en Uruguay; 2014.

16. Callejo Gallego J. Observación, entrevista y grupo de discusión: el silencio de tres prácticas de investigación. Rev Esp Salud Pública 2002; 76:409-22.

17. Aran D, Locan H. Sistema de salud en Uruguay. Salud Pública Méx 2011; 53:265-74.

18. Santos J, Tambellini C, Oliveira S. Reflexión sobre la presencia del acompañante durante el proceso de parto. REME Rev Min Enferm 2011; 15:453-8. 
19. Castro R. Teoría social y salud. México DF: Editorial Lugar; 2011.

20. Erviti J. Construcción de los objetos profesionales, orden corporal y desigualdad social. Una reflexión en torno a las interacciones médicosusuarios de servicios ginecológicos. In: López A, Castro R, editores. Poder médico y ciudadanía: el conflicto social de los profesionales de la salud con los derechos reproductivos en América Latina. Avances y desafíos en la investigación regional. Montevideo: Cátedra Libre en Salud Reproductiva, Sexualidad y Género, Facultad de Psicología, Universidad de la República/Centro Regional de Investigaciones Multidisciplinarias, Universidad Nacional Autónoma de México; 2010. p. 101-22.

21. Perinat A. Psicología del desarrollo: un enfoque sistémico. Barcelona: Editorial UOC; 2010.

22. Ministerio de Salud Pública. Ordenanza Ministerial no 217/09. Norma Nacional de Lactancia Materna. Montevideo: Ministerio de Salud Pública; 2009.

23. Olza Fernández I, Lebrero E. ¿Nacer por cesárea? Bogotá: Grupo Editorial Norma; 2006.

24. Indriunas F, Lourenzi A. Bonilha a participação do pai como acompanhante da mulher no parto. Texto Contexto Enferm 2011; 20:44552.

25. Blanca J, Abalos M, Montes M, González S El papel del padre en el periodo del posparto: experiencias con el método piel con piel. Acta Paul Enferm 2012; 25:915-20.

26. Villalón H, Toro R, Riesco I, Pinto M, Silva C. Participación paterna en la experiencia del parto. Rev Chil Pediatr 2014; 85:554-60.

27. Santos J, Tambellini C, Oliveira S. Reflexión sobre la presencia del acompañante durante el proceso de parto. REME Rev Min Enferm 2011; 15:453-58.
28. Olza Fernández I. El trastorno de estrés postraumático como secuela obstétrica. Cuadernos de Medicina Psicosomática y Psiquiatria de Enlace 2010; (96):35-41

29. Caires TLG, Vargens OMC. A exclusão do pai da sala de parto: uma discussão de género e poder. Referência 2012; III:159-68.

30. Aguilar Cordero MJ, Sáez Martín I, Menor Rodríguez MJ, Mur Villar N, Expósito Ruiz M, Hervás Pérez A, et al. Satisfaction rating in a group of women from Granada on birthing care, support and breastfeeding lenght. Nutr Hosp 2013; 28:920-6.

31. Beretta M, Zaneti D, Fabbro M, de Freitas M, Ruggiero E, Dupas G. Tristeza/depressão na mulher: uma abordagem no período gestacional e/ou puerperal. Rev Eletrônica Enferm 2008; 10:966-78.

32. Montoya DIG, González Mazuelo EM, Henao López CP. Experiencias de las mujeres durante el trabajo de parto y parto. Avances en Enfermería 2015; 33:271-81.

33. Salehi A, Fahami F, Beigi M. The effect of presence of trained husbands beside their wives during childbirth on women's anxiety. Iran J Nurs Midwifery Res 2016; 21:611-5. 


\section{Abstract}

The article aims to analyze how women are accompanied by a trusted person during cesareans, as guaranteed under Law 17,386 on "accompanying persons during labor and childbirth", enacted in Uruguay in 2001. The findings are part of a larger study on the experience and meaning of cesareans for Uruguayan women. An exploratory, descriptive qualitative methodology was used for this purpose. The technique involved indepth interviews with 31 women whose cesareans were performed in their first pregnancy in the city and greater metropolitan area of Montevideo. Based on the findings, the conclusion is that having a trusted accompanying person during labor and childbirth is experienced by women as a factor for emotional protection given the anxieties generated by a major surgery like a cesarean. Nevertheless, for the women interviewed in the study, "being accompanied" was not a continuous process, but characterized by a series of separations, both from the accompanying persons and from their children, causing anxiety, anguish, ambivalent feelings towards the newborn infant, and difficulties in mother-infant bonding, especially in the immediate postpartum.

Reproductive Health; Cesarean Section; Obstetric Labor

\section{Resumo}

O objetivo deste artigo é analisar o acompanhamento durante a cesariana, por parte de uma pessoa de confiança da mulher, consignado pela Lei no 17.386 de "parto assistido", adotada no Uruguai no ano de 2001. Os resultados fazem parte de uma pesquisa maior sobre a experiência e o significado da cesariana para as mulheres que passaram por esta experiência. Para esse fim, foi escolhida uma metodologia qualitativa de caráter exploratório e descritivo. A técnica utilizada foi a entrevista en profundidade, realizada em um total de 31 mulheres, cujas cesarianas tiveram lugar na sua primeira gestação, em Montevidéu e área metropolitana (Uruguai). A partir dos resultados obtidos, concluiu-se que o acompanhamento no processo de trabalho de parto e nascimento é experimentado pelas mulheres como um fator de proteção emocional ante as ansiedades que geram a experiência de uma cirurgia maior como a cesariana. No entanto, para as mulheres entrevistadas, o "acompanhamento" ao invés de continuo foi caracterizado por uma série de separações, tanto de seus acompanhantes, como de seus filhos/as, o qual provocou ansiedade, aflição, sentimentos de ambivalência em relação ao recém-nascido, dificuldades no estabelecimento do vínculo materno, especialmente no puerpério imediato.

Saúde Reprodutiva; Cesárea; Trabalho de Parto

Recibido el 13/Oct/2016

Versión final presentada el 28/Ene/2017

Aprobado el 25/Abr/2017 